\title{
Rice Nitrogen Status Estimation of Western tract of Odisha using SVM based on color feature: A comparative Analysis with LCC
}

$* 1$ Prabira Kumar Sethy, ${ }^{2}$ Bibhuti Bhusan Nayak, ${ }^{3}$ Nalini Kanta Barpanda, ${ }^{4}$ Amiya Kumar Rath

${ }^{1,2,3}$ Department of Electronics, Sambalpur University, Burla, India-768019

${ }^{4}$ Department of Computer Science and Engineering, VSSUT Burla, India-768017

Email:prabirsethy.05@gmail.coml, bibhiti.bhusan.nayak@suiit.ac.in ${ }^{2}$,

nkbarpanda@suniv.ac.in ${ }^{3}$,amiyaamiya@ rediffmail.com ${ }^{4}$

\begin{abstract}
Rice is the most important human food crop in the world, directly feeding more people than any other crop. Rice crop contains different types of nutrients. Sometime due to deficiency of nutrition, rice crops undergo in a hindrance effects on its growth and production. Nitrogen is the main component among all nutrients for rice crop growth and production. The leaf nitrogen concentration (LNC) is highly correlated with chlorophyll content. There are many devices like Leaf Color Chart (LCC), SPAD, at LEAF+ for measurement of chlorophyll \&/ or nitrogen. As these devices are cost effective and unavailable with all farmers, a digitize image acquisition and interpretation system is required. This paper proposed a site-specific approach for nitrogen status estimation based on image captured by smart phone, matching with LCC and train the support vector machine (SVM) using radial basis function (RBF). The proposed methodology have accuracy of 98\%, which implies it is quite successful to classify the rice leaf according to their nitrogen status.
\end{abstract}

Keywords- LCC; Rice Nitrogen Status; Support Vector Machine; Radial Basis Function.

\section{INTRODUCTION}

Rice is life, for most people living in Odisha. Rice has shaped the cultures, diets and economies of thousands of millions of people. In precision agriculture, it is needful to estimate the nitrogen status of rice crop for healthy growth and large production. The nitrogen content of most Indian soil varies from 0.03 to 0.07 percent as against 0.1 to 0.17 percent in Europe and American soil (T.J. Mirchandani. 1936 [1]). Nitrogen is the nutrient, which limits the most the rice production worldwide. In Asia, where more than 90 percent of the world's rice produced and about 60 percent of the $\mathrm{N}$ fertilizer used on rice (Stangel and De Dutta, 1985[2]). Leaf Color Chart (LCC) is a standard chart to assess leaf color level according to its nitrogen status. It was develop by the International Rice Research Institute (IRRI) for monitoring the status of nitrogen level by matching the color of rice leaf with the swap of LCC. LCC has five green strips, with color ranging from yellow green to dark green. It determines the greenness of the rice leaf, which indicates its $\mathrm{N}$ content [3]. The fig. 1 depicts the Leaf color chart (LCC) developed by Central Rice Research Institute (CRRI-ICAR) Cuttack. To perform the observation, it need two aspects i.e. (1) good color perception of observer, (2) observe consistently throughout the crop growth. Even though there is availability of some instruments and use by many researcher but not popular among farmer. According to statistic the number of mobile phone user from 2013 to 2019 is consistently increase. The statistic in Fig. 2 shows the number of mobile phone users in India from 2013 to 2019. For 2017, the number of mobile phone users in India expected to rise to 730.7 million. In this same year, the number of smartphone users in India predicted to reach 340 million and could reach almost 468 million by 2021 [4].

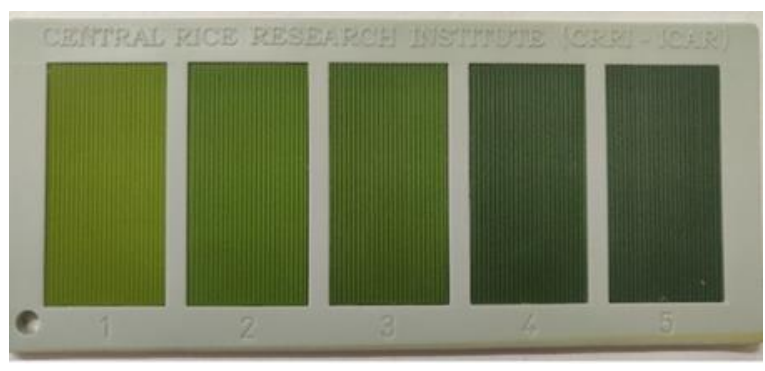

Fig.1. Leaf Color Chart developed by Central Rice Research Institute (CRRI-ICAR), Cuttack.

Considering the prevalent utilization of cell phones among people, this innovation will utilized to capture, analyse and estimate the leaf color with our proposed procedure. 


\section{International Journal of Research in Advent Technology, Vol.7, No.4, April 2019 \\ E-ISSN: 2321-9637 \\ Available online at www.ijrat.org}

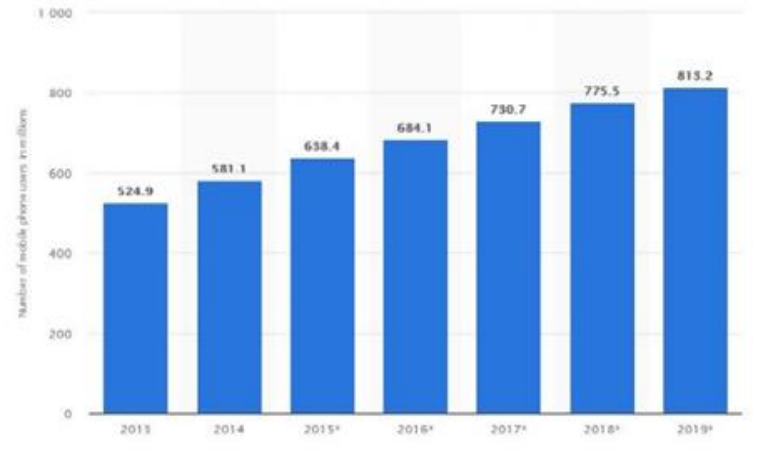

Fig.2.The statistic of the number of mobile phone users in India from 2013 to 2019.

Several researcher reported various methodology for estimating the nitrogen status of rice crop as well as other crops. Yuita Arum Sari et al.2013 [5] proposed a method of relative color calibration, which makes the system, learns color chart automatically without depending on specific standard color. K- NearestNeighbour (KNN) classification used for color learning process in RGB color space. The method successfully tested with two smartphone devices in different lighting condition. The test shows an average accuracy above the threshold value of $83 \%$. Shah Jahan Leghari et al.2016 [6] designed a modern mirror made LCC named Nitrom and claim that it is stronger against sunlight and no sectional color can reduce its brightness and thus does not become dim in color due to adverse effect of heat or light, because, its mirror functioning as protection from all environmental factors including sunlight and precipitation. I. Wayan Astika 2010 [7] proposed a color level estimation of rice leaf using smart phone camera in two lighting condition i.e. body shadow and open sunlight. The KNN used for classification purpose of different color level, validated with LCC, and achieve $66 \%$ in body shadow \& $68 \%$ in open sunlight. Mohd. Soom et al. 2013 [8] used Tetracam Agriculture Digital Camera to acquire high spatial and temporal resolution images to determine the status of $\mathrm{N}$ and predict the grain yield of rice. They use 12 pots of rice plant with nitrogen treatment. The image samples were collected in different growing stages and achieved coefficient of correlation for normalized difference vegetative index NDVI i.e. $\mathrm{R}^{2}$ is 0.78. Yuan Wang. 2014 [9] proposed a methodology to estimate the chlorophyll content and leaf nitrogen concentration (LNC) using 13 number of color indices with three 3 color space and validated with SPAD. The author claim that the La*b* color model is more correlated with the SPAD measurement. Mohammad Mehdi Saberioon. et al.
2013 [10] proposed assessment of color indices using digital camera for estimating nitrogen status. Here the color indices derived from images captured by two different kinds of conventional digital cameras and extracted twenty-seven most common color indices through six growth stages. The results indicated that both cameras could use as sensors to determine the status of nitrogen in rice plants. In addition, there were strong relationships between most indices, especially color indices that are associated with green and SPAD measurement values.

\section{MATERIAL AND METHODOLOGY}

The methodology consists of four parts i.e. first is to collect the sample of rice leaf, second part is to extract the RGB color channel then the third part is determine the value of mean $R$, mean $G$, mean $B$ and summation of these three mean. By using these four feature SVM, having RBF kernel function trained and estimate the test sample according to its nitrogen status, illustrated in figure 3 .

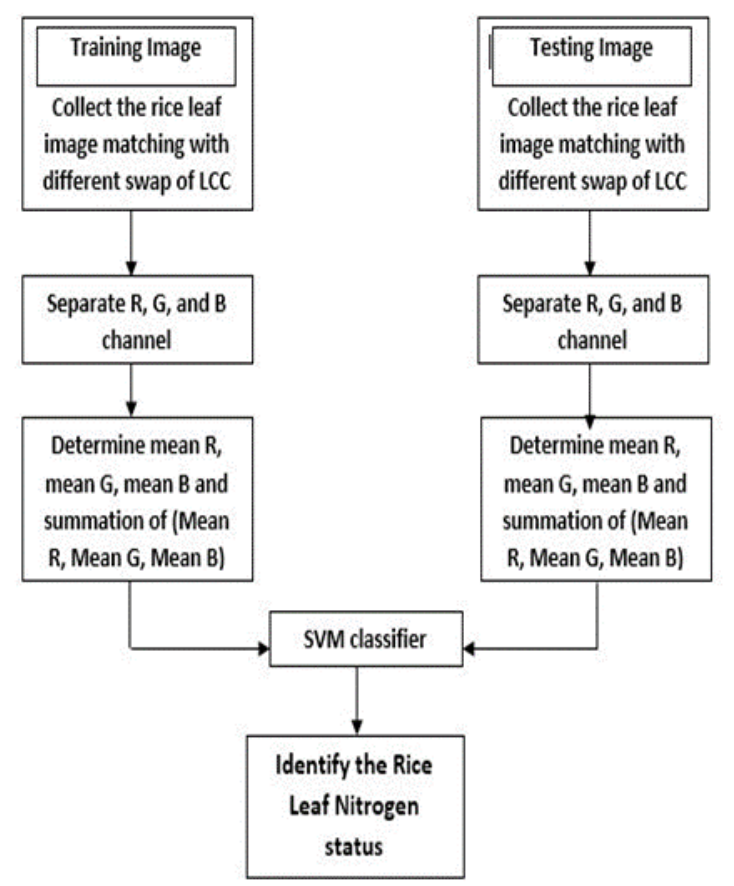

Fig.3 Framework of nitrogen status estimation of Rice Leaf.

\subsection{Collection of sample}

The images were captured using phone of 12 Megapixel camera from five different places of 


\section{E-ISSN: 2321-9637}

\section{Available online at www.ijrat.org}

western tract of Odisha i.e.Attabira (longitude:83.7748,latitude:21.3717), Dhanakauda (longitude:84.1627,latitude:20.968730),Saranda(longit ude:86.386612,latitude:22.266991),Attabira(longitude: 3.782660,latitude: 21.371950) and Bargarh (longitude:83.643300, latitude).

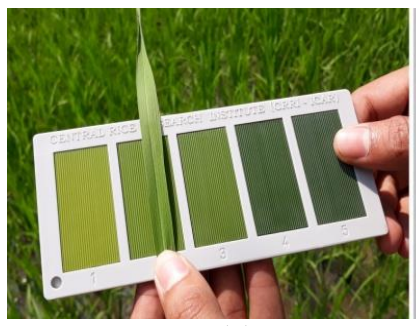

(a)

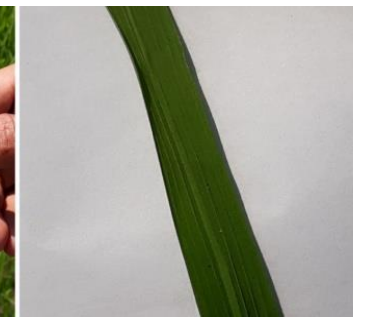

(b)
Fig.4. Collection of image sample (a) Testing of rice leaf using LCC (b) photo shoot of rice leaf using smart phone camera with white back ground

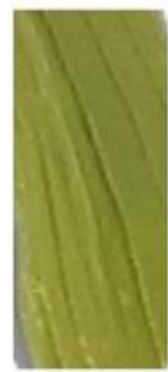

(a)

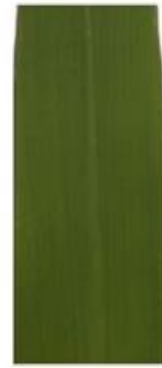

(b)

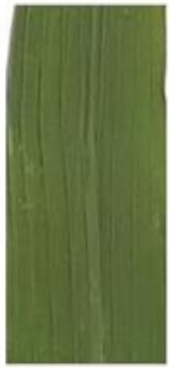

(c)

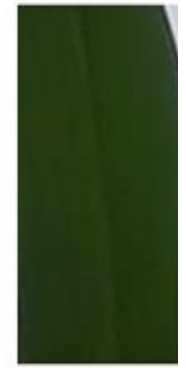

(d)
Fig.5. Rice Leaf of different Swap (a) Swap 1, (b) Swap 2, (c) Swap 3, (d) Swap 4.

The procedure of collection of sample illustrated in figure 4.There are five type of swap is present in LCC but as per the availability of nitrogen in soil of western Odisha up to fourth level of nitrogen are present. Therefore, we took the four types swap and limited our study with ignorance of fifth swap. According to report of G.C. Sahu et al. 2003 [11] the western Odisha especially Sambalpur and Bargarh district have laterite, mixed red and yellow red soil. In Sambalpur district, the soil is laterite soil, which contain very less nitrogen. Again the mixed red and yellow soil are present in both Sambalpur and Bargarh district in which the upland soil have low nitrogen $\&$ phosphorus and low land have low nitrogen \& medium phosphorus.

\subsection{Feature Extraction}

LCC was develop by IRRI (International Rice Research Institute) and by FRRI (Philippines Rice Research Institute). In India, these charts provided by Nitrogen parameters, which provide guidance to the farmers regarding improved Nitrogen (N) management. Nitrogen applied by the farmers in rice plants at different growth stages, but the amount of $\mathrm{N}$ to be apply and the time of applications vary substantially. LCC helps to synchronize $\mathrm{N}$ application with the real time and quantity demand of the rice crop. Helps to prevent under or over usage of $\mathrm{N}$ in rice plants. The LCC is collected from IRRI, Cuttack by cost of 120INR. Then we collect the sample of rice leaf of different swap matching with the LCC. The image of 20 number of leaf sample of each swap are capture and extract four texture feature such as mean of $\mathrm{R}$, mean of $\mathrm{G}$, mean of $\mathrm{B}$ and summation of mean $\mathrm{R}$, mean $\mathrm{G}$ \&mean $\mathrm{B}$.

\subsection{Classification}

Support vector machines are supervised learning models with an associated learning algorithm that analyses data used for classification. SVM is popular for classification than other classifier as it can separate linear as well as non-linear space (the kernel trick). The other uniqueness of SVM is that it is less overfitting and work with smaller data. Radial basis functions are means to approximate multivariable (also called multivariate) functions by linear combinations of terms based on a single univariate function (the radial basis function). This is radialised so that in can be used in more than one dimension. They are usually apply to approximate functions or data, which are known at a finite number of points (or too difficult to evaluate otherwise), so that then evaluations of the approximating function can take place often and efficiently. The radial basis function kernel is a popular and powerful kernel used in pattern recognition and illustrated in Eq. (1).

$$
G\left(X_{1}-X_{2}\right)=\exp \left(-|| X_{1}-X_{2}||\right)^{2} \quad \text { Eq. (1) }
$$

\section{RESULT AND DISCUSSION}

The methodology is examine by use of 10 number of each sample image of four variety (matching with swap1 to swap 4). Then the four texture feature are extracted i.e. mean $\mathrm{R}$, mean $\mathrm{G}$, mean $\mathrm{B}$ and summation of mean $R$, mean $G \&$ mean $B$. It is observed that, the range of mean $\mathrm{R}$ for swap 1, swap2, swap3 and swap 4 are (125.38 to 146.8992), (13.6438 to 80.7109$), 94.61868$ to 110.0957$)$ and (28.66503 to 40.47361) respectively. Again the range of mean $\mathrm{G}$ for swap1, swap2, swap 3 and swap4 are (139.807 to 159.101), (89.30243 to 91.67421), 109.6587to 


\section{International Journal of Research in Advent Technology, Vol.7, No.4, April 2019 \\ E-ISSN: 2321-9637 \\ Available online at www.ijrat.org}

134.3342) and (49.64154 to 64.32703) respectively. The range of mean B of swap 1 to 4 are (46.9102 to $72.088)$, (31.339 to 43.58402$)$, (57.11632 to 69.62775) and (12.14162 to 15.58505$)$. In addition, the sum parameter, which is summation of mean R, mean G \& mean B of swap1 to 4 are (313.8827 to 334.7259$)$, (191.7124 to 213.923$)$, (261.3937 to 314.9567$)$ and (101.2577 to 314.2577$)$ respectively. It is notice that there is some intersection between some texture parameter in between two swap like mean R of swap1 $\&$ swap2 and sum parameter of swap4 \& swap1. That is why we use RBF kernel of SVM to classify the nitrogen status of rice leaf, as the feature are nonlinear in nature. Again, for testing purpose we use 80 number of rice leaf sample of 20 number of each variety (from swap1 to swap 4 matching with LCC). According to performance analysis of proposed methodology its shown that all swap are predicted correctly except swap4. In case of swap4 out 20 number of sample, 2 are predicted as swap2. So in total out of 80 sample 78 correctly predicted. Therefore, the overall accuracy of proposed methodology is $98 \%$.

Table 1. Comparative analysis of proposed methodology with LCC.

\begin{tabular}{|l|l|l|l|l|}
\hline & Swap-1 & Swap-2 & Swap-3 & Swap-4 \\
\hline Algorithm & 20 & 20 & 20 & 18 \\
\hline LCC & 20 & 20 & 20 & 20 \\
\hline
\end{tabular}

\section{CONCLUSION}

The proposed methodology for nitrogen status estimation by SVM using radial basis function kernel is an ultimatum for farmer. As now a days all most all civilian including rural people are use smart phone having in-built camera. So this methodology may a preliminary step to develop android \&/or web based application to estimate the nitrogen status of rice leaf. This methodology having accuracy of $98 \%$ by considering four swap from swap1 to swap4. The limitation of this work is that, in the work we are not considering swap 5, which is for high concentration of nitrogen because of non-availability in western Odisha.

\section{REFERENCES}

[1] T.J. Mirchandani. 1936. Nitrogen Status of Indian Soil. Symposium on Nitrogen Supply to Indian Soil.
[2] Stangel, P. J. and De Dutta, S. K. (1985). Availability of inorganic fertilizers and their management - a focus on Asia. IRRI conference, 1-5 June 1985. IRRI, Manila, Philippines.

[3] http://www.knowledgebank.irri.org.

[4] www.statista.com.

[5] Yuita Arum Sari, R V Hari Ginardi, Riyanarto Sarno, "Assessment of Color Levels in Leaf Color Chart Using Smartphone Camera with Relative Calibration Information Systems" (2013). International Conference - ISICO, Denpasar, Bali.

[6] Shah Jahan Leghari, Umed Ali Leghari, Mahmooda Buriro, Ghulam Mustafa Laghari, Farooque Ahmed Soomro, Maqsood Ahmed Khaskheli, Syed Sadam Hussain. 2016. Modern Leaf Colour Chart Successfully Prepared and Used in Crop Production of Sindh, Pakistan EUROPEAN ACADEMIC RESEARCH - Vol. IV (2).pp.900-916.

[7] I. Wayan Astika, "The Use of Hand Phone Camera to Determine Paddy Leaf Color Level as Reference for Feritilizing Dosage”, AFITA 2010 International Conference, The Quality Information for Competitive Agricultural Based Production System and Commerce, 2010.

[8] Mohd Soom, Mohd Amin and Wayayok, Aimrun and Abdul Rahim, Anuar and Saberioon, Mohammadmehdiand Gholizadeh,

Asa (2013) Multi-Spectral Images Tetracam agriculture Digital Camera to Estimate Nitrogen and Grain Yield of Rice at difference Growth Stages. The Philippine agriculturist, 96 (1). pp. 108-112. ISSN 0031-7454.

[9] Yuan Wang et al. 2014. Estimating rice chlorophyll content and leaf nitrogen concentration with a digital still color camera under natural light. Plant Methods. pp. 2-11.

[10] Mohammad Mehdi Saberioon. et al. 2013. Assessment of colour indices derived from conventional digital camera for determining nitrogen status in rice plants. Journal of Food, Agriculture \& Environment Vol.11 (2): 655-662.

[11] G.C. Sahu and Antaryami Mishra. Soil of Odisha and its Management. Odisha Review 2005.

[12] A. Chaudhuri, K. De and D. Chatterjee, "A Comparative Study of Kernels for the Multi-class Support Vector Machine," 2008 Fourth International Conference on Natural Computation, Jinan, 2008, pp. 3-7.doi: 10.1109/ICNC.2008.803.

[13] Paul F. Evangelista et al., " Some Properties of the Gaussian Kernel for One Class Learning", international conference on Artificial Neural Networks, ICANN07 Porto, Portugal, September 2007. 